\title{
Editorial: Past, Present, and Future Impacts of Climate on Infrastructure
}

\author{
Abhishek Gaur ${ }^{1 *}$ and Ronita Bardhan ${ }^{2,3}$ \\ ${ }^{1}$ National Research Council Canada, Ottawa, ON, Canada, ${ }^{2}$ Department of Architecture, University of Cambridge, \\ Cambridge, United Kingdom, ${ }^{3}$ Centre for Urban Science and Engineering, Indian Institute of Technology Bombay, Mumbai, \\ India
}

Keywords: climate change, infrastructures, resiliency, sustainability, natural disasters

\section{Editorial on the Research Topic}

\section{Past, Present, and Future Impacts of Climate on Infrastructure}

Climate change is one of the biggest challenges that the global community faces. The changing climate may lead to the infrastructure bring exposed to unprecedented climate with an increase in the frequency and intensity of extreme weather events, such as more intense rain events and flooding, extreme winds, landslides, and other hazards, that could result in infrastructure damage and failure (Stocker et al., 2013). The consequences of failure can be quite significant and cause fatalities, injuries, and illnesses, disruption or loss of service, increased costs to infrastructure owners, and unforeseen costs to infrastructure users, and considerable negative socioeconomic impacts to the governments.

Infrastructure systems are primarily located in urban areas. The urban climate is often different from the surrounding rural climate. It is generally warmer, rainier, less windy, and more polluted. This means that more drastic effects of changing climate will be experienced by the urban infrastructure systems than the surrounding areas (Krayenhoff et al., 2018). The cities and infrastructure systems will also be overburdened in the future due to ongoing rapid urbanization. It is predicted that by the 2050 s, $66 \%$ of the world's population will live in urban areas, up from about $50 \%$ living in the urban areas in the year 2007, making the infrastructure systems increasingly strained in the future also due to increases in urban population (UN, 2014).

To design urban infrastructure systems considering the non-stationarity in climate, it is essential to assess the impacts of past, current, and future climate on the infrastructure systems. This will entail developing approaches to reliably model the extreme climate hazards and their interactions with the complex urban systems. The papers part of this Research Topic aims to provide new knowledge in these areas.

Saha and Ghosh study the relative impacts of future projected climate and land-use change on the hydrological response of the Ganga river basin in India. The complex chain of analysis performed included: generation of future climate projections following different global warming scenarios and socioeconomic pathways, preparation of future land-use scenarios using a land allocation model and performing hydrologic simulations using a semi-distributed hydrologic model, followed by application of Bodyko framework to understand the relative impacts of climate and land-use changes on the basin characteristics. The study found that as a consequence of global warming, the Ganga river basin will become more arid in the future. However, the basin's future hydrologic response will mostly be governed by projected changes in climate. Land-use changes will have minimal effect on its hydrologic response.

Yan et al. provided a review of a recently developed science-driven engineering product: next-generation Intensity-Duration-Frequency (NG-IDF) curve to establish a consistent IDF design methodology for both rain-dominated and snow-dominated regions. The NG-IDF captures multiple flood-generating mechanisms, including rainfall, snowmelt, and rain-on-snow 
as opposed to the typical precipitation-based IDF curves (PRECIDF), which only captures flood occurrences due to extreme rainfall. NG-IDF is the outcome of a coordinated effort from climate scientists developing necessary climate information with global and regional scale climate models, hydrologists simulating snow processes and estimating water available for runoff using hydrologic models, the civil engineering community on integrating the snow processes into the IDF design process. Recent developments toward validating the NG-IDF curves on a larger spatiotemporal domain and incorporating future projected effects of climate change more accurately in them are discussed.

Bondank and Chester advocate that infrastructure systems and not merely complicated systems that contain many parts and there is uncertainty included in the system, they are complex systems characterized by "unpredictability and the presence of unknown unknowns," and so the common cause-and-effect approach of managing the uncertainty of the failure of infrastructure systems in the face of climate change hazards may not be best suited to model them. They recommend that best practices from complex system sciences such as Decision Making Under Deep Uncertainty and Safe-to-Fail frameworks should be used to improve the decision-making when managing the complex infrastructure systems. Besides, it is highlighted that the communication and coordination between managers of different infrastructure systems need to be enhanced to better implement strategies.

Data is central in the climate change debate. Especially data that is multidimensional and explores the societal impacts are crucial for informed decision making. Using information as evidence to derive social vulnerability is much needed. Barankin et al. describe this in their work on an evidencedriven approach for assessing social vulnerability during extreme events. A novel data-driven predictive approach is forwarded that overcomes over-generalization or aggregation in the indicatorbased method. Using the case of Hurricane Sandy in the State of New Jersey, the authors demonstrate variability in the vulnerability among the Minorities" is substantial, with a low approval rate in the insurance claims. The study successfully showed that using the need-based, evidence-driven method

\section{REFERENCES}

Krayenhoff, E. S., Moustaoui, M., Broadbent, A. M., Gupta, V., and Georgescu, M. (2018). Diurnal interaction between urban expansion, climate change and adaptation in US cities. Nat. Clim. Change 8, 1097-1103. doi: 10.1038/s41558-018-0320-9

Stocker, T. F., Qin, D., Plattner, G.-K., Tignor, S. K., Allen, J., Boschung, A., et al. (2013). Climate Change 2013: The Physical Science Basis. Working Group 1 (WG1) Contribution to the Intergovernmental Panel on Climate Change (IPCC) 5th Assessment Report (AR5). New York, NY; Cambridge.

UN (2014). World Urbanization Prospects: The 2014 Revision, Highlights (ST/ESA/SER.A/352). Department of Economic and Social Affairs, Population Division. New York, NY. provides a validation route for vulnerability assessments and is scalable across geographies. The universality of the process is worth reproducing. It can be considered the new direction of research on climate-related vulnerability measurements unbiased from the statistical inflation of indicators.

Markolf et al., while exploring the opportunities and challenges for artificial intelligence applications in infrastructure management to combat climate change, emphasizes that handling rapid technological transitions is the primary challenge. The magnanimity and the complexity of the problem make it incognisable for any individual or organization to handle. Artificial intelligence offers a seamless ability to manage complexity while providing insightful feedback. Although the authors underscore that while AI provides potential benefits which outweighs the drawbacks of over-reliance on reliable data, they are cautious in mentioning that an open dialogue is required.

A similar understanding is put forward by Nawroz Tonmoy et al., where the potential of utilizing smart city frameworks for disaster resilience in coastal cities is reviewed. The authors use a unique infrastructural lens to review the academic literature that focuses on smart systems' new development in coastal disaster management. The findings are interesting as they point out that while IoT and crisis informatics offer considerable potential for disaster resiliency, it remains understudied for coastal cities which need disaster resiliency that their inland counterparts.

The topic editorial team of this Research Topic on Past, Present, and Future Impacts of Climate on Infrastructure would like to thank all of the authors for considering this Research Topic for submitting their scholarly work. Thanks to the reviewers' hard work who provided their expert reviews under very tight schedules, the quality of the final papers presented in this Research Topic have dramatically improved. Without their contributions, this Research Topic would not have been so timely and successful.

\section{AUTHOR CONTRIBUTIONS}

AG and RB wrote sections of the manuscript. Both authors contributed equally to the manuscript revision, read, and approved the submitted version.
Conflict of Interest: The authors declare that the research was conducted in the absence of any commercial or financial relationships that could be construed as a potential conflict of interest.

Copyright (c) 2021 Gaur and Bardhan. This is an open-access article distributed under the terms of the Creative Commons Attribution License (CC BY). The use, distribution or reproduction in other forums is permitted, provided the original author(s) and the copyright owner(s) are credited and that the original publication in this journal is cited, in accordance with accepted academic practice. No use, distribution or reproduction is permitted which does not comply with these terms. 